\title{
On the statistics of the minimal solution of a linear Diophantine equation and uniform distribution of the real part of orbits in hyperbolic spaces
}

\author{
Morten S. Risager and Zeév Rudnick
}

\begin{abstract}
We study a variant of a problem considered by Dinaburg and Sinal̆ on the statistics of the minimal solution to a linear Diophantine equation. We show that the signed ratio between the Euclidean norms of the minimal solution and the coefficient vector is uniformly distributed modulo one. We reduce the problem to an equidistribution theorem of Anton Good concerning the orbits of a point in the upper half-plane under the action of a Fuchsian group.
\end{abstract}

\section{Statement of results}

1.1. For a pair of coprime integers $(a, b)$, the linear Diophantine equation $a x-b y=1$ is well known to have infinitely many integer solutions $(x, y)$, any two differing by an integer multiple of $(b, a)$. Dinaburg and Sinar [2] studied the statistics of the "minimal" such solution $v^{\prime}=\left(x_{0}, y_{0}\right)$ when the coefficient vector $v=(a, b)$ varies over all primitive integer vectors lying in a large box with commensurate sides. Their notion of "minimality" was in terms of the $L^{\infty}$-norm $\left|v^{\prime}\right|_{\infty}:=\max \left(\left|x_{0}\right|,\left|y_{0}\right|\right)$, and they studied the ratio $\left|v^{\prime}\right|_{\infty} /|v|_{\infty}$, showing that it is uniformly distributed in the unit interval. Other proofs were subsequently given by Fujii [5] who reduced the problem to one about modular inverses, and then used exponential sum methods, in particular a non-trivial bound on Kloosterman sums, and by Dolgopyat [3, who used continued fractions.

In this note, we consider a variant of the question by using minimality with respect to the Euclidean norm $|(x, y)|^{2}:=x^{2}+y^{2}$ and study the ratio $\left|v^{\prime}\right| /|v|$ of the Euclidean norms as the coefficient vector varies over a large ball. In this case too we find uniform distribution, in the interval $[0,1 / 2]$. However, the methods involved appear quite different, as we invoke an equidistribution theorem of Anton Good [8] which uses harmonic analysis on the modular curve.

2000 Mathematics Subject Classification. Primary 11J71; Secondary 11M36.

The first author was funded by a Steno Research Grant from The Danish Natural Science Research Council. The second author was supported by the Israel Science Foundation (grant No. $925 / 06)$. 
1.2. A lattice point problem. We recast the problem in slightly more general and geometric terms. Let $L \subset \mathbb{C}$ be a lattice in the plane, and let area $(L)$ be the area of a fundamental domain for $L$. Any primitive vector $v$ in $L$ can be completed to a basis $\left\{v, v^{\prime}\right\}$ of $L$. The vector $v^{\prime}$ is unique up to a sign change and addition of a multiple of $v$. In the case of the standard lattice $\mathbb{Z}[\sqrt{-1}]$, taking $v=(a, b)$ and $v^{\prime}=(x, y)$, the condition that $v, v^{\prime}$ give a basis of $\mathbb{Z}[\sqrt{-1}]$ is equivalent to requiring $a y-b x= \pm 1$. The question is: If we pick $v^{\prime}$ to minimize the length $\left|v^{\prime}\right|$ as we go through all possible completions, how does the ratio $\left|v^{\prime}\right| /|v|$ between the lengths of $v^{\prime}$ and $v$ fluctuate? It is easy to see (and we will prove it below) that the ratio is bounded, indeed that for a minimizer $v^{\prime}$ we have

$$
\frac{\left|v^{\prime}\right|}{|v|} \leq \frac{1}{2}+O\left(\frac{1}{|v|^{4}}\right) \text {. }
$$

We will show that the ratio $\left|v^{\prime}\right| /|v|$ is uniformly distributed in $[0,1 / 2]$ as $v$ ranges over all primitive vectors of $L$ in a large (Euclidean) ball.

We refine the problem slightly by requiring that the lattice basis $\left\{v, v^{\prime}\right\}$ is oriented positively, that is $\operatorname{Im}\left(v^{\prime} / v\right)>0$. Then $v^{\prime}$ is unique up to addition of an integer multiple of $v$. For the standard lattice $\mathbb{Z}[\sqrt{-1}]$ and $v=(a, b), v^{\prime}=(x, y)$ the requirement is then that $a y-b x=+1$. Define the signed ratio by

$$
\rho(v):= \pm\left|v^{\prime}\right| /|v|
$$

where we chose $\left|v^{\prime}\right|$ minimal, and the sign is + if the angle between $v$ and $v^{\prime}$ is acute, and - otherwise.

Theorem 1.1. As $v$ ranges over all primitive vectors in the lattice $L$, the signed ratio $\rho(v)$ is uniformly distributed modulo one.

Explicitly, let $L_{\text {prim }}(T)$ be the set of primitive vectors in $L$ of norm $|v| \leq T$. It is well known that

$$
\# L_{\text {prim }}(T) \sim \frac{1}{\zeta(2)} \frac{\pi}{\operatorname{area}(L)} T^{2}, \quad T \rightarrow \infty
$$

Theorem 1.1 states that for any fixed subinterval $[\alpha, \beta] \in(-1 / 2,1 / 2]$,

$$
\frac{1}{\# L_{\text {prim }}(T)}\left\{v \in L_{\text {prim }}(T): \alpha<\rho(v)<\beta\right\} \rightarrow \beta-\alpha
$$

as $T \rightarrow \infty$.

1.3. Equidistribution of real parts of orbits. We will reduce Theorem 1.1 by geometric arguments to a result of Anton Good [8] on uniform distribution of the orbits of a point in the upper half-plane under the action of a Fuchsian group.

Let $\Gamma$ be discrete, co-finite, non-cocompact subgroup of $\mathrm{SL}_{2}(\mathbb{R})$. The group $\mathrm{SL}_{2}(\mathbb{R})$ acts on the upper half-plane $\mathbb{H}=\{z \in \mathbb{C}: \operatorname{Im}(z)>0\}$ by linear fractional transformations. We may assume, possibly after conjugation in $\mathrm{SL}_{2}(\mathbb{R})$, that $\infty$ is a cusp and that the stabilizer $\Gamma_{\infty}$ of $\infty$ in $\Gamma$ is generated by

$$
\pm\left(\begin{array}{ll}
1 & 1 \\
0 & 1
\end{array}\right)
$$

which as linear fractional transformation gives the unit translation $z \mapsto z+1$. (If $-I \notin \Gamma$ there should be no \pm in front of the matrix). The group $\Gamma=\mathrm{SL}_{2}(\mathbb{Z})$ is an example of such a group. We note that the imaginary part of $\gamma(z)$ is fixed on the 
orbit $\Gamma_{\infty} \gamma z$, and that the real part modulo one is also fixed on this orbit. Good's theorem is

Theorem $1.2(\operatorname{Good}[\mathbf{8})$. Let $\Gamma$ be as above and let $z \in \mathbb{H}$. Then $\operatorname{Re}(\Gamma z)$ is uniformly distributed modulo one as $\operatorname{Im}(\gamma z) \rightarrow 0$.

More precisely, let

$$
\left(\Gamma_{\infty} \backslash \Gamma\right)_{\varepsilon, z}=\left\{\gamma \in \Gamma_{\infty} \backslash \Gamma: \operatorname{Im} \gamma z>\varepsilon\right\} .
$$

Then for every continuous function $f \in C(\mathbb{R} / \mathbb{Z})$, as $\varepsilon \rightarrow 0$,

$$
\frac{1}{\#\left(\Gamma_{\infty} \backslash \Gamma\right)_{\varepsilon, z}} \sum_{\gamma \in\left(\Gamma_{\infty} \mid \Gamma\right)_{\varepsilon, z}} f(\operatorname{Re} \gamma z) \rightarrow \int_{\mathbb{R} / \mathbb{Z}} f(t) d t
$$

Though the writing in $[\mathbf{8}$ is not easy to penetrate, the results deserve to be more widely known. We sketch a proof of Theorem 1.2 in appendix $\mathrm{A}$ assuming familiarity with standard methods of the spectral theory of automorphic forms.

Acknowledgements: We thank Peter Sarnak for his comments on an earlier version and for alerting us to Good's work.

\section{A geometric argument}

2.1. We start with a basis $\left\{v, v^{\prime}\right\}$ for the lattice $L$ which is oriented positively, that is $\operatorname{Im}\left(v^{\prime} / v\right)>0$. For a given $v, v^{\prime}$ is unique up to addition of an integer multiple of $v$. Consider the parallelogram $P\left(v, v^{\prime}\right)$ spanned by $v$ and $v^{\prime}$. Since $\left\{v, v^{\prime}\right\}$ form a basis of the lattice $L, P\left(v, v^{\prime}\right)$ is a fundamental domain for the lattice and the area of $P\left(v, v^{\prime}\right)$ depends only on $L$, not on $v$ and $v^{\prime}$ : area $\left(P\left(v, v^{\prime}\right)\right)=\operatorname{area}(L)$.

Let $\mu(L)>0$ be the minimal length of a nonzero vector in $L$ :

$$
\mu(L)=\min \{|v|: 0 \neq v \in L\} .
$$

Lemma 2.1. Any minimal vector $v^{\prime}$ satisfies

$$
\left|v^{\prime}\right|^{2} \leq\left(\frac{|v|}{2}\right)^{2}+\left(\frac{\operatorname{area}(L)}{|v|}\right)^{2} .
$$

Moreover, if $|v|>2$ area $(L) / \mu(L)$ then the minimal vector $v^{\prime}$ is unique up to sign.

Proof. To see (2.1), note that the height of the parallelogram $P$ spanned by $v$ and $v^{\prime}$ is area $(P) /|v|=\operatorname{area}(L) /|v|$. If $h$ is the height vector, then the vector $v^{\prime}$ thus lies on the affine line $h+\mathbb{R} v$ so is of the form $h+t v$. After adding an integer multiple of $v$ we may assume that $|t| \leq 1 / 2$, a choice that minimizes $\left|v^{\prime}\right|$, and then

$$
\left|v^{\prime}\right|^{2}=t^{2}|v|^{2}+|h|^{2} \leq \frac{1}{4}|v|^{2}+\left(\frac{\operatorname{area}(L)}{|v|}\right)^{2} .
$$

We now show that for $|v| \gg_{L} 1$, the minimal choice of $v^{\prime}$ is unique if we assume $\operatorname{Im}\left(v^{\prime} / v\right)>0$, and up to sign otherwise: Indeed, writing the minimal $v^{\prime}$ as above in the form $v^{\prime}=h+t v$ with $|t| \leq 1 / 2$, the choice of $t$ is unique unless we can take $t=1 / 2$, in which case we have the two choices $v^{\prime}=h \pm v / 2$. To see that $t= \pm 1 / 2$ cannot occur for $|v|$ sufficiently large, we argue that if $v^{\prime}=h+v / 2$ then we must have $2 h=2 v^{\prime}-v \in L$. The length of the nonzero vector $2 h$ must then be at least $\mu(L)$. Since $|h|=\operatorname{area}(L) /|v|$ this gives $2 \operatorname{area}(L) /|v| \geq \mu(L)$, that is

$$
|v| \leq \frac{2 \operatorname{area}(L)}{\mu(L)}
$$

Hence $v^{\prime}$ is uniquely determined if $|v|>2$ area $(L) / \mu(L)$. 
2.2. Let $\alpha=\alpha_{v, v^{\prime}}$ be the angle between $v$ and $v^{\prime}$, which takes values between 0 and $\pi$ since $\operatorname{Im}\left(v^{\prime} / v\right)>0$. As is easily seen, for any choice of $v^{\prime}, \sin \alpha_{v, v^{\prime}} \operatorname{shrinks}$ as we increase $|v|$, in fact we have:

Lemma 2.2. For any choice of $v^{\prime}$ we have

$$
\sin \alpha \leq \frac{\operatorname{area}(L)}{\mu(L)} \frac{1}{|v|} .
$$

Proof. To see (2.2), note that the area of the fundamental parallelogram $P\left(v, v^{\prime}\right)$ is given in terms of $\alpha$ and the side lengths by

$$
\operatorname{area}(P)=|v|\left|v^{\prime}\right| \sin \alpha
$$

and since $v^{\prime}$ is a non-zero vector of $L$, we necessarily have $\left|v^{\prime}\right| \geq \mu(L)$ and hence, since $\operatorname{area}(P)=\operatorname{area}(L)$ is independent of $v$,

$$
0<\sin \alpha \leq \frac{\operatorname{area}(L)}{\mu(L)|v|}
$$

as claimed.

Note that if we take for $v^{\prime}$ with minimal length, then we have a lower bound $\sin \alpha \geq 2$ area $(L) /|v|^{2}+O\left(1 /|v|^{6}\right)$ obtained by inserting (2.1) into the area formula $\operatorname{area}(L)=|v|\left|v^{\prime}\right| \sin \alpha$.

2.3. Given a positive basis $\left\{v, v^{\prime}\right\}$, we define a measure of skewness of the fundamental parallelogram as follows: Let $\Pi_{v}\left(v^{\prime}\right)$ be the orthogonal projection of the vector $v^{\prime}$ to the line through $v$. It is a scalar multiple of $v$ :

$$
\Pi_{v}\left(v^{\prime}\right)=\operatorname{sk}\left(v, v^{\prime}\right) v
$$

where the multiplier $\operatorname{sk}\left(v, v^{\prime}\right)$, which we call the skewness of the parallelogram, is given in terms of the inner product between $v$ and $v^{\prime}$ as

$$
\operatorname{sk}\left(v, v^{\prime}\right)=\frac{\left\langle v^{\prime}, v\right\rangle}{|v|^{2}} .
$$

Thus we see that the skewness is the real part of the ratio $v^{\prime} / v$ :

$$
\operatorname{sk}\left(v, v^{\prime}\right)=\operatorname{Re}\left(v^{\prime} / v\right) .
$$

If we replace $v^{\prime}$ by adding to it an integer multiple of $v$, then $\operatorname{sk}\left(v, v^{\prime}\right)$ changes by

$$
\operatorname{sk}\left(v, v^{\prime}+n v\right)=\operatorname{sk}\left(v, v^{\prime}\right)+n
$$

In particular, since $v^{\prime}$ is unique up to addition of an integer multiple of $v$, looking at the fractional part, that is in $\mathbb{R} / \mathbb{Z}$, we get a quantity $\operatorname{sk}(v) \in(-1 / 2,1 / 2]$ depending only on $v$ :

$$
\operatorname{sk}(v):=\operatorname{sk}\left(v, v^{\prime}\right) \quad \bmod 1 .
$$

This is the least skewness of a fundamental domain for the lattice constructed from the primitive vector $v$.

Lemma 2.3. The signed ratio $\rho(v)= \pm\left|v^{\prime}\right| /|v|$ and the least skewness $\operatorname{sk}(v)$ are asymptotically equivalent:

$$
\rho(v)=\operatorname{sk}(v)\left(1+O\left(\frac{1}{|v|^{2}}\right)\right) .
$$


Proof. In terms of the angle $0<\alpha<\pi$ between the vectors $v$ and $v^{\prime}$, we have

$$
\operatorname{sk}\left(v, v^{\prime}\right)=\frac{\left|v^{\prime}\right|}{|v|} \cos \alpha \text {. }
$$

Our claim follows from this and the fact $\cos \alpha= \pm 1+O\left(1 /|v|^{2}\right)$, which follows from the upper bound (2.2) of Lemma 2.2

Thus the sequences $\{\rho(v)\},\{\operatorname{sk}(v)\}$ are asymptotically identical, hence uniform distribution of one implies that of the other. To prove Theorem 1.1 it suffices to show

Theorem 2.4. As $v$ ranges over all primitive vectors in the lattice $L$, the least skewness $\operatorname{sk}(v)$ become uniformly distributed modulo one.

This result, for the standard lattice $\mathbb{Z}[\sqrt{-1}]$, was highlighted by Good in the introduction to 8 . Below we review the reduction of Theorem 2.4 to Theorem 1.2

2.4. Proof of Theorem 2.4. Our problems only depend on the lattice $L$ up to scaling. So we may assume that $L$ has a basis $L=\{1, z\}$ with $z=x+i y$ in the upper half-plane. The area of a fundamental domain for $L$ is area $(L)=\operatorname{Im}(z)$. Any primitive vector has the form $v=c z+d$ with the integers $(c, d)$ co-prime.

Now given the positive lattice basis $v=c z+d$ and $v^{\prime}=a z+b$, form the integer matrix $\gamma=\left(\begin{array}{ll}a & b \\ c & d\end{array}\right)$, which has $\operatorname{det}(\gamma)=+1$ since $\left\{v, v^{\prime}\right\}$ form a positive basis of the lattice. Thus we get a matrix in the modular group $\Gamma=S L_{2}(\mathbb{Z})$. Then with $\gamma$ applied as a Möbius transformation to $z$, the length of $v$ can be computed via

$$
\operatorname{Im}(\gamma z)=\frac{\operatorname{Im}(z)}{|c z+d|^{2}}=\frac{\operatorname{area}(L)}{|v|^{2}}
$$

The signed ratio between the lengths of $v$ and $v^{\prime}$ (when $v^{\prime}$ is chosen of minimal length) is

$$
\rho(v)= \pm|\gamma z| .
$$

where the sign is + if $\operatorname{Re}(\gamma z)>0$ and - otherwise. Moreover, we have

$$
\operatorname{sk}\left(v, v^{\prime}\right)=\operatorname{Re}(\gamma z)
$$

Indeed,

$$
\operatorname{Re}(\gamma z)=\frac{a c\left(x^{2}+y^{2}\right)+(a d+b c) x+b d}{|c z+d|^{2}}
$$

which is $\operatorname{sk}\left(v, v^{\prime}\right)$ in view of (2.3). Consequently, the uniform distribution modulo one of $\operatorname{sk}(v)$ as $|v| \rightarrow \infty$ is then exactly the uniform distribution modulo one of $\operatorname{Re}(\gamma z)$ as $\gamma$ varies over $\Gamma_{\infty} \backslash \Gamma$ with $\operatorname{Im}(\gamma z) \rightarrow 0$, that is Theorem 1.2

\section{Appendix A. A sketch of a proof of Good's theorem}

To prove Theorem 1.2 we use Weyl's criterion to reduce it to showing that the corresponding "Weyl sums" satisfy

$$
\sum_{\gamma \in\left(\Gamma_{\infty} \mid \Gamma\right)_{\varepsilon, z}} e(m \operatorname{Re} \gamma z)=\delta_{m=0} \frac{t_{\Gamma}}{\operatorname{vol}(\Gamma \backslash \mathbb{H})} \frac{1}{\varepsilon}+o(1 / \varepsilon)
$$


as $\varepsilon \rightarrow 0$. Here $t_{\Gamma}$ equals 2 if $-I \in \Gamma$ and 1 otherwise. In turn, (A.1) will follow, by a more or less standard Tauberian theorem (see e.g. [11, p. 1035-1038]) from knowing the analytic properties of the series

$$
V_{m}(z, s):=\sum_{\gamma \in \Gamma_{\infty} \mid \Gamma} \operatorname{Im}(\gamma z)^{s} e(m \operatorname{Re}(\gamma z)) .
$$

studied also in [7, 10] Here $e(x)=\exp (2 \pi i x)$. The series is absolutely convergent for $\operatorname{Re}(s)>1$, as is seen by comparison with the standard non-holomorphic Eisenstein series $V_{0}(z, s)=E(z, s)$ of weight 0 (See [16]). For general $m$ the series is closely related to the Poincaré series

$$
U_{m}(z, s)=\sum_{\gamma \in \Gamma_{\infty} \mid \Gamma} \operatorname{Im}(\gamma z)^{s} e(m \gamma z)
$$

studied by Selberg 15. For a different application of the series $V_{m}(z, s)$, see $\mathbf{1 3}$.

The analytic properties from which we can conclude Theorem 1.2 are given by

Proposition A.1. The series $V_{m}(z, s)$ admits meromorphic continuation to $\operatorname{Re}(s)>$ $1 / 2$. If poles exist they are real and simple. If $m \neq 0$ then $V_{m}(z, s)$ is regular at $s=1$. If $m=0$ the point $s=1$ is a pole with residue $t_{\Gamma} / \operatorname{vol}(\Gamma \backslash \mathbb{H})$. Moreover, $V_{m}(z, s)$ has polynomial growth on vertical strips in $\operatorname{Re}(s)>1 / 2$.

Sketch of proof. The claim about continuation of $V_{0}(z, s)=E(z, s)$ is well-known and goes back to Roelcke [12] and Selberg [14. To handle also $m \neq 0$ we may adopt the argument of Colin de Verdière [1, Théorème 3] and of Goldfeld and Sarnak [6] to get the result. This is done as follows: Consider the hyperbolic Laplacian

$$
\Delta=-y^{2}\left(\frac{\partial^{2}}{\partial x^{2}}+\frac{\partial^{2}}{\partial y^{2}}\right) \text {. }
$$

If we restrict $\Delta$ to smooth functions on $\Gamma \backslash \mathbb{H}$ which are compactly supported it defines an essentially self-adjoint operator on $L^{2}(\Gamma \backslash \mathbb{H}, d \mu)$ where $d \mu(z)=d x d y / y^{2}$, with inner product

$$
\langle f, g\rangle=\int_{\Gamma \backslash \mathbb{H}} f(z) \overline{g(z)} d \mu(z) .
$$

We will also denote by $\Delta$ the self-adjoint closure.

Let $h(y)$ be a smooth function which equals 0 if $y<T$ and 1 if $y>T+1$ where $T$ is sufficiently large. One may check that when $\operatorname{Re}(s)>1$

$$
V_{m}(z, s)-h(y) y^{s} e(m x)
$$

is square integrable. This is an easy exercise using [9, Theorem 2.1.2]. The series $V_{m}(z, s)$ satisfies

$$
(\Delta-s(1-s)) V_{m}(z, s)=(2 \pi m)^{2} V_{m}(z, s+2) \text { when } \operatorname{Re}(s)>1,
$$

since $f_{s}(z)=y^{s} e^{2 \pi i m \operatorname{Re} z}$ satisfies this equation and because the Laplacian commutes with isometries, so does $V_{m}(z, s)$, being a sum of translates of $f_{s}$. Therefore

$$
\begin{aligned}
& (\Delta-s(1-s))\left(V_{m}(z, s)-h(y) y^{s} e(m x)\right) \\
& =(2 \pi m)^{2}\left(V_{m}(z, s+2)-h(y) y^{s+2} e(m x)\right) \\
& \quad-h^{\prime \prime}(y) y^{s+2} e(m x)-2 h^{\prime}(y) y^{s+1} e(m x)
\end{aligned}
$$


is also square integrable, since the last two terms are compactly supported. We can therefore use the resolvent $(\Delta-s(1-s))^{-1}$ to invert this and find

$$
V_{m}(z, s)-h(y) y^{s} e(m x)=(\Delta-s(1-s))^{-1}\left((2 \pi m)^{2} V_{m}(z, s+2)-H(z, s)\right)
$$

where

$$
\left.H(z, s)=(2 \pi m)^{2} h(y) y^{s+2} e(m x)\right)+h^{\prime \prime}(y) y^{s+2} e(m x)+2 h^{\prime}(y) y^{s+1} e(m x)
$$

This defines the meromorphic continuation of $V_{m}(z, s)$ to $\operatorname{Re}(s)>1 / 2$ by the meromorphicity of the resolvent (see e.g [4]). The singular points are simple and contained in the set of $s \in \mathbb{C}$ such that $s(1-s)$ is an eigenvalue of $\Delta$. Since $\Delta$ is self-adjoint, these lie on the real line $($ when $\operatorname{Re}(s)>1 / 2)$. The potential pole at $s=1$ has residue a constant times

$$
\int_{\Gamma \backslash \mathbb{H}}(2 \pi m)^{2} V_{m}(z, 3)-H(z, 1) d \mu
$$

The contribution from $h^{\prime \prime}(y) y^{s+2} e(m x)+2 h^{\prime}(y) y^{s+1} e(m x)$ is easily seen to be zero if $T$ is large enough using $\int_{0}^{1} e(m x) d x=0$ when $m \neq 0$. To handle the rest we may unfold to get

$$
\begin{aligned}
(2 \pi m)^{2} & \int_{\Gamma \backslash \mathbb{H}}\left(V_{m}(z, 3)-h(y) y^{3} e(m x)\right) d \mu(z) \\
& =(2 \pi m)^{2} \int_{0}^{\infty} \int_{0}^{1}\left(y^{3}-h(y) y^{3}\right) e(m x) y^{-2} d x d y=0
\end{aligned}
$$

so $V_{m}(z, s)$ is analytic at $s=1$. The claim about growth in vertical strips is proved as in [11, Lemma 3.1].

Remark A.2. It is possible to extend the main idea of the proof of Proposition A.1 to prove the meromorphic continuation of $V_{m}(z, s)$ to $s \in \mathbb{C}$. But since our main aim was to prove Theorem 1.2 we shall stop here.

\section{References}

[1] Yves Colin de Verdière. Pseudo-laplaciens. II. Ann. Inst. Fourier (Grenoble), 33(2):87-113, 1983.

[2] Efim I. Dinaburg and Yakov G. Sinal. The statistics of the solutions of the integer equation $a x-b y= \pm 1$. Funktsional. Anal. $i$ Prilozhen., 24(3):1-8, 96, 1990.

[3] Dimitry Dolgopyat. On the distribution of the minimal solution of a linear Diophantine equation with random coefficients. Funktsional. Anal. i Prilozhen., 28(3):22-34, 95, 1994.

[4] Ludvig D. Faddeev. The eigenfunction expansion of Laplace's operator on the fundamental domain of a discrete group on the Lobačevskiı̌ plane. Trudy Moskov. Mat. Ob̌šc., 17:323-350, 1967.

[5] Akio Fujii. On a problem of Dinaburg and Sină. Proc. Japan Acad. Ser. A Math. Sci., 68(7):198-203, 1992.

[6] Dorian Goldfeld and Peter Sarnak. Sums of Kloosterman sums. Invent. Math., 71(2):243-250, 1983.

[7] A. Good. Beiträge zur Theorie der Dirichletreihen, die Spitzenformen zugeordnet sind. J. Number Theory, 13(1):18-65, 1981.

[8] Anton Good. On various means involving the fourier coefficients of cusp forms. Mathematische Zeitschrift, 183(1):95-129, 1983.

[9] Tomio Kubota. Elementary theory of Eisenstein series. Kodansha Ltd., Tokyo, 1973.

[10] H. Neunhöffer. Über die analytische Fortsetzung von Poincaréreihen. S.-B. Heidelberger Akad. Wiss. Math.-Natur. Kl., pages 33-90, 1973.

[11] Yiannis N. Petridis and Morten S. Risager. Modular symbols have a normal distribution. Geom. Funct. Anal., 14(5):1013-1043, 2004. 
[12] Walter Roelcke. Analytische Fortsetzung der Eisensteinreihen zu den parabolischen Spitzen von Grenzkreisgruppen erster Art. Math. Ann., 132:121-129, 1956.

[13] Peter Sarnak. Estimates for Rankin-Selberg $L$-functions and quantum unique ergodicity. $J$. Funct. Anal., 184(2):419-453, 2001.

[14] Atle Selberg. Discontinuous groups and harmonic analysis. In Proc. Internat. Congr. Mathematicians (Stockholm, 1962), pages 177-189. Inst. Mittag-Leffler, Djursholm, 1963.

[15] Atle Selberg. On the estimation of Fourier coefficients of modular forms. In Proc. Sympos. Pure Math., Vol. VIII, pages 1-15. Amer. Math. Soc., Providence, R.I., 1965.

[16] Atle Selberg. Göttingen lecture notes in Collected papers. Vol. I. Springer-Verlag, Berlin, 1989. With a foreword by K. Chandrasekharan.

Department of Mathematical Sciences, University of Aarhus, Ny Munkegade BuildING 530, 8000 Aarhus C, Denmark

E-mail address: risager@imf.au.dk

School of Mathematical Sciences, Tel Aviv University, Tel Aviv 69978, Israel

E-mail address: rudnick@post.tau.ac.il 